\title{
The Geological Disaster Monitoring Method Based on GNSS
}

\author{
Cai $\mathrm{Qi}^{1, \mathrm{a}}$, Jiang Jian ${ }^{1, b}$, Chen Wei ${ }^{2, c}$ \\ ${ }^{1}$ School of Chemical Engineering and Environment, Beijing Institute of Technology, Beijing 100081, \\ China; \\ ${ }^{2}$ Chinese Academy of Science Institute of Automation, Beijing 100190, China. \\ accaiqibit@163.com, 'bjw202@bit.edn.cn, 'csales@21lensys.com
}

Keywords: GNSS, geological disaster, relative position, monitoring method, cloud computing.

\begin{abstract}
In recent years, the frequent occurrence of geological disasters such as landslides, debris flow and so on causes huge losses to the lives and property of the people. To solve this problem, this paper proposes a geological disaster monitoring method based on GNSS (Global Navigation Satellite System), and the relative motion state of the monitoring points is calculated by relative positioning algorithm and coordinate transformation. The actual running data of the Chongqing geological disaster monitoring project shows that the method can effectively monitor the geological movement, and the user can obtain the information of the monitoring points conveniently and quickly through the cloud technology.
\end{abstract}

\section{Introduction}

In recent years, geological disasters have occurred frequently in China, which has caused huge losses to the lives and property of the people. In our country, the main types of geological disasters include landslides, debris flow, land collapse and so on. These disasters will have a clear omen. As long as the disasters can be discovered in time, it will be able to greatly reduce the loss. Therefore, an effective method of the geological disaster monitoring is particularly important. Many geological disasters occur after the obvious relative displacement of the surface and the relative displacement of the monitoring points can effectively reflect the geological movement, so as to provide a reliable basis for the geological disaster warning.

GNSS has the advantages that other traditional sensors do not have, such as high precision, all-weather, no accumulated error and so on [1]. Most of the monitoring sites are prone to geological disasters and not suitable for human monitoring, so it is urgent to a remote monitoring method free of maintenance. In view of the problem of the geological disaster monitoring, this paper presents a method of monitoring the relative displacement of the monitoring points by relative positioning algorithm and coordinate transformation based on GNSS data. The method is used for the monitoring of geological disasters in Chongqing. The results show that the method can effectively monitor the geological disasters. The relative position changes of the monitoring points can be remotely monitored by cloud technology so that people can avoid monitoring on the spot and personal safety can be protected. The method has high value of engineering use.

\section{Solution of GNSS positioning data}

The Monitoring Method Based On GNSS. For the geological disaster monitoring method, the most important is to monitor the relative displacement of the monitoring points prone to disasters in real time. The monitoring method based on GNSS, through the installation in stable geological conditions for a base station satellite antenna and the installation where prone to geological disasters for the measurement station satellite antenna, is real-time access to the ephemeris information. A baseline attitude can be obtained through the solution of every two antennas' ephemeris information [2]. So the relative motion of the measuring station can be obtained by solving the position of the station relative to the base station. This information is then stored in the database for the later analysis. At the same time, the information about the relative position of the measurement station will display 
on the Webpage to the observers. When it changes more than the limit, the monitoring personnel can timely warn so that the nearby residents can leave in time. Figure 1 is the schematic diagram of GNSS monitoring method.

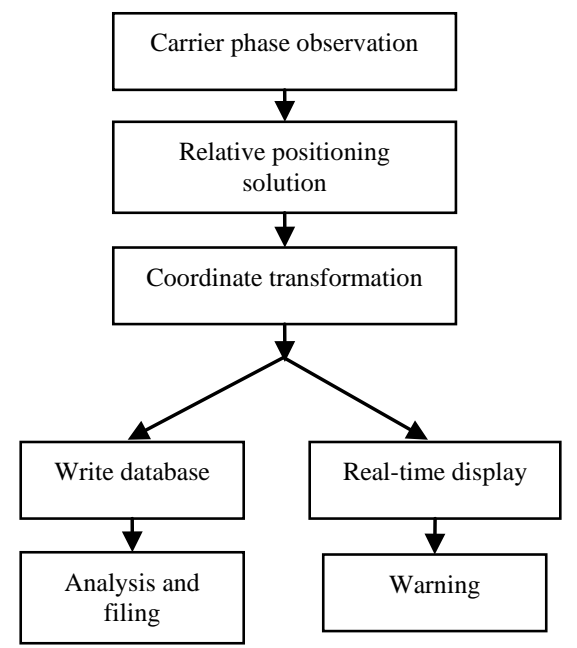

Fig. 1 The schematic diagram of GNSS monitoring method

The receiver used in the paper is NovAtel dual frequency GNSS receiver which can receive ephemeris information from GPS and GLONASS satellite navigation system and can improve the calculation accuracy and speed [3].

Principle of the Baseline Attitude Solution. In order to obtain the relative position of the monitoring points, the position of the measuring station relative to the base station is measured but not the absolute position. So this paper chose to use relative localization algorithm.

According to the observation data obtained by a satellite, the observed equation can be obtained:

$(N+\varphi) \lambda=R+c \cdot \tau+\sigma$.

In the formula, $\mathrm{N}$ is the phase integer ambiguity, $\varphi$ is the phase fraction, $\lambda$ is the carrier wavelength, $\mathrm{R}$ is the actual distance between the antenna and the satellite, $\tau$ is the receiver clock error, $\sigma$ is the delay error correction for the satellite signal through the troposphere and ionosphere.

The satellite is about $20000 \mathrm{~km}$ away from the antenna, so the path of the signal to the two measuring point can be considered the same. In other words, the ionosphere and troposphere delay of the same satellite signal to the two observation point are equal [4]. To make difference between the two satellite measurements and the observation of the same time, we can eliminate the errors caused by the ionosphere and troposphere delay, and can also eliminate the orbit error and the satellite clock error of the same satellite. Then we can get the single difference equation [5]:

$$
\left(\nabla N_{a b}^{j}-\nabla \varphi_{a b}^{j}\right) \lambda=\nabla R_{a b}^{j}+c\left(\tau_{a}-\tau_{b}\right)
$$

In the formula, $\nabla N_{a b}^{j}=\nabla N_{a}^{j}-\nabla N_{b}^{j}$ is the difference between the phase integers of the two antennas to the same satellite at the same time and $\nabla \varphi_{a b}^{j}=\nabla \varphi_{a}^{j}-\nabla \varphi_{b}^{j}$ is the difference between the phase fractions, $\nabla R_{a b}^{j}=\nabla R_{a}^{j}-\nabla R_{b}^{j}$ is the difference between the actual distances, $c\left(\tau_{a}-\tau_{b}\right)$ is the difference between the receiver clock errors.

The clock in the satellite antenna receiver is generally $10^{-6} \mathrm{~s}$. In the calculation, the absolute clock error of the receiver is difficult to estimate [6]. Therefore, to make difference between the formulas obtained by the two antennas to two satellites at the same time. Then we can get the double difference equation [7]:

$\left(\Delta \nabla N_{a b}^{j k}-\Delta \nabla \varphi_{a b}^{j k}\right) \lambda=\Delta \nabla R_{a b}^{j k}$

Then the equation (3) is linearized. By assuming that the approximation of the baseline vector $\mathrm{B}$ is $\left(\Delta X_{a b}^{0}, \Delta Y_{a b}^{0}, \Delta Z_{a b}^{0}\right)^{T}$ and the correction is $\left(\delta X_{a b}, \delta Y_{a b}, \delta Z_{a b}\right)^{T}$, the approximation of the phase integer ambiguity is $\left(N_{a b}^{j k}\right)_{0}$ and the correction is $\delta N_{a b}^{j k}$, we can get the error equation [8]:

$$
V_{a b}^{j k}=\alpha_{a b}^{j k} \delta V_{a b}^{j k}+\beta_{a b}^{j k} \delta V_{a b}^{j k}+\gamma_{a b}^{j k} \delta V_{a b}^{j k}+\delta N_{a b}^{j k}
$$


In the formula,

$$
\begin{aligned}
& \alpha_{a b}^{j k}=\frac{1}{2 \lambda}\left(\frac{\Delta X_{a}^{j}}{R_{a}^{j}}+\frac{\Delta X_{b}^{j}}{R_{b}^{j}}-\frac{\Delta X_{a}^{k}}{R_{a}^{k}}-\frac{\Delta X_{b}^{k}}{R_{b}^{k}}\right) ; \\
& \beta_{a b}^{j k}=\frac{1}{2 \lambda}\left(\frac{\Delta Y_{a}^{j}}{R_{a}^{j}}+\frac{\Delta Y_{b}^{j}}{R_{b}^{j}}-\frac{\Delta Y_{a}^{k}}{R_{a}^{k}}-\frac{\Delta Y_{b}^{k}}{R_{b}^{k}}\right) ; \\
& \gamma_{a b}^{j k}=\frac{1}{2 \lambda}\left(\frac{\Delta Z_{a}^{j}}{R_{a}^{j}}+\frac{\Delta Z_{b}^{j}}{R_{b}^{j}}-\frac{\Delta Z_{a}^{k}}{R_{a}^{k}}-\frac{\Delta Z_{b}^{k}}{R_{b}^{k}}\right) ; \\
& W_{a b}^{j k}=\alpha_{a b}^{j k} \Delta X_{a b}^{0}+\beta_{a b}^{j k} \Delta Y_{a b}^{0}+\gamma_{a b}^{j k} \Delta Z_{a b}^{0}+\left(N_{a b}^{j k}\right)_{0}-\Delta \nabla \varphi_{a b}^{j k} 。
\end{aligned}
$$

If there are $n$ satellites, we could get $n-1$ error equations. Then $n-1$ carrier phase integer ambiguities are need. The number of variables is $(n-1)+3$. By observing p epochs, we can get $p(n-1)$ error equations. Thus we get redundant observations that could be solved with least the squares principle [9]. The error equation can be put into matrix form:

$V=A \cdot X+L$

In the formula, $V=\left(V_{1}, V_{2}, \ldots, V_{n}\right)^{T} ; X=\left(\delta X, \delta Y, \delta Z, \delta N_{1}, \delta N_{2}, \ldots, \delta N_{n}\right)^{T} ; L=\left(W_{1}, W_{2}, \ldots, W_{n}\right)^{T}$; The $\mathrm{A}$ is the coefficient matrix. By assuming that each double difference is equal and independent, we can get the equation:

$$
N \cdot X+D=0
$$

In the formula, $N=A^{T} A ; D=A^{T} L$ 。

Then we can get $X=-N^{-1} D=-\left(A^{T} A\right)^{-1}\left(A^{T} L\right)$. The length of the baseline and its components are:

$$
\begin{aligned}
& L=\sqrt{\Delta X_{a b}^{2}+\Delta Y_{a b}^{2}+\Delta Z_{a b}^{2}} \\
& \Delta X_{a b}=\Delta X_{a b}^{0}+\delta X_{a b} \\
& \Delta Y_{a b}=\Delta Y_{a b}^{0}+\delta Y_{a b} \\
& \Delta Z_{a b}=\Delta Z_{a b}^{0}+\delta Z_{a b}
\end{aligned}
$$

For the reliability of the results, this paper introduces the whole week search factor called the ratio. The ratio is defined as the ratio between the second minimum value of the posterior variance and the minimum value of the posterior variance [10]. The greater the value of the ratio is, the more reliable the results are. In this paper, the critical value of ratio is 3 . If the ratio value is greater than 3 , the result is reliable. Otherwise, the result should be removed.

\section{Coordinate transformation and data application}

After the solution, the baseline attitude vector $\left(\Delta X_{a b}, \Delta Y_{a b}, \Delta Z_{a b}\right)^{T}$ is based on earth-centered earth-fixed (ECEF) coordinate system. In order to represent the posture of the baseline better, we need to carry out the transformation from ECEF to Earth-fixed coordinate system (ENU). For the moving object on the surface of the Earth, its East, North, and Up coordinate components in the ENU system are more meaningful in physics than its $\mathrm{X}, \mathrm{Y}$ and $\mathrm{Z}$ coordinate components in the ECEF system [11].

The transformation relationship between ECEF and ENU is as below: $(\Delta E, \Delta N, \Delta U)^{T}=S \cdot(\Delta X, \Delta Y, \Delta Z)^{T}$

In the formula,

$$
S=\left[\begin{array}{c}
-\sin \lambda \\
-\sin \phi \cos \lambda \\
\cos \phi \cos \lambda
\end{array}\right.
$$

$\cos \lambda$
$-\sin \phi \sin \lambda$
$\cos \phi \sin \lambda$

$\left.\begin{array}{c}0 \\ \cos \phi \\ \sin \phi\end{array}\right]$

the longitude of the antenna [12]. 
By real-time calculation of the relative changes about ENU of the measurement station to the base station, the geological conditions of monitoring points can be obtained and geological disaster warning can be carried on reliably.

\section{Result analysis}

The data used in this paper is the actual data of the Chongqing geological disaster monitoring site. The site is equipped with a base station and a measurement station. The base line is composed of the two stations. In the analysis, the mean value of each half hour represents the half hour data.

Figure 2 is Chongqing geological disaster monitoring data curve from July 8th 0 AM to July 9th 0 AM.
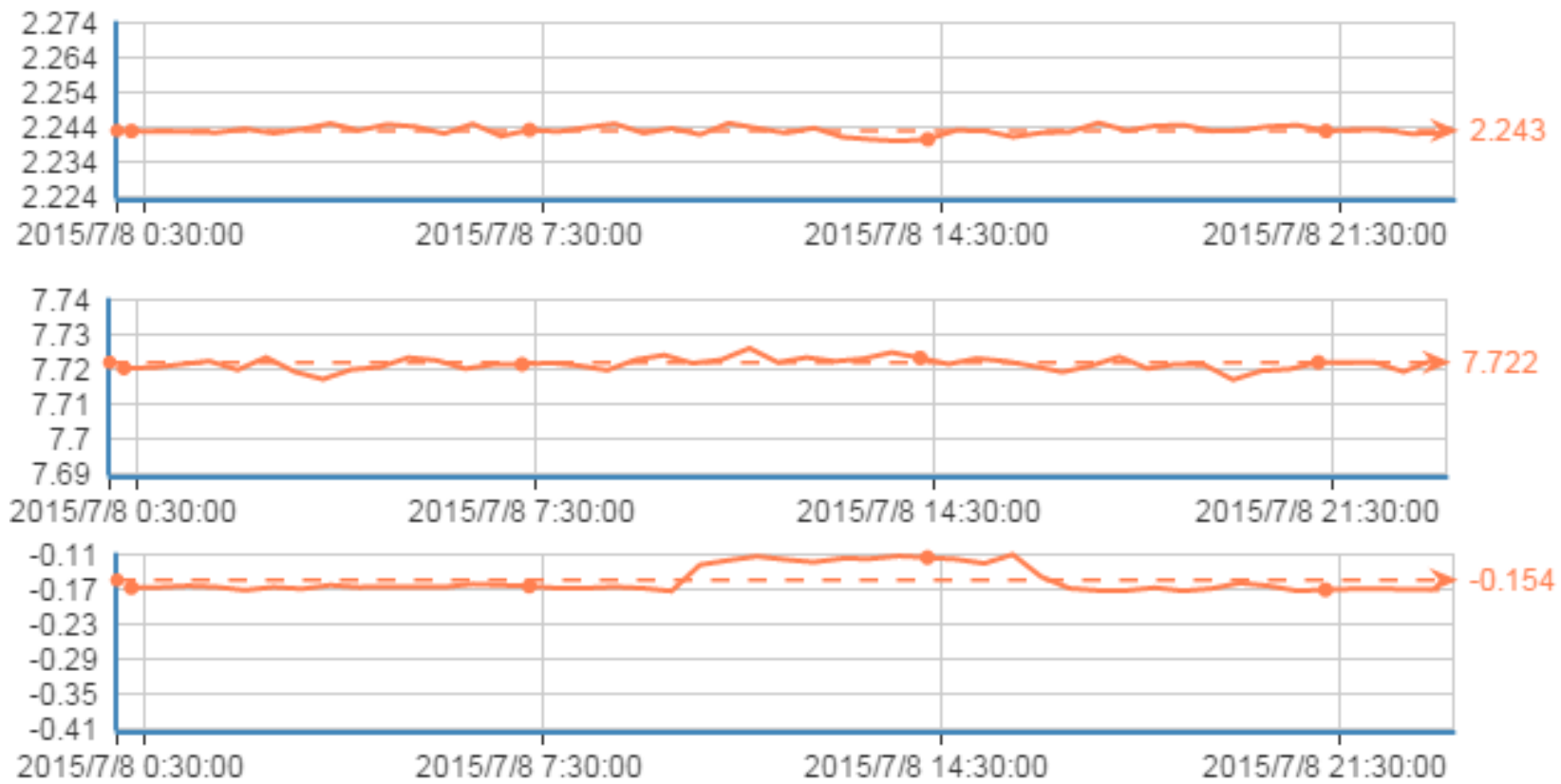

Fig. 2 Operation data

In Figure 2, the $\mathrm{X}$ axis is working time; the $\mathrm{Y}$ axis in the first picture is the East coordinate component; The $\mathrm{Y}$ axis in the second picture is the North coordinate component; the $\mathrm{Y}$ axis in the third picture is the Up coordinate component. The field test person raised the measurement station 50mm in the Up direction in 10:00:00 and recovered it in 16:00:00. From the data, we can see that when the monitoring point changes the position, the measurement curve can reflect the change well and the error is controlled within $1 \mathrm{~cm}$. The result is as Table 1 . The unit is millimeter.

Table 1 Comparison between the real change and measurement change

\begin{tabular}{cccc}
\hline Time & change for $\mathrm{E}$ & change for $\mathrm{N}$ & Change for $\mathrm{U}$ \\
\hline 10:00:00(real) & 0 & 0 & 50 \\
10:00:00(measurement) & -1.80 & -2.36 & 52.91 \\
16:00:00(real) & 0 & 0 & -50 \\
16:00:00(measurement) & 1.17 & -1.43 & -58.25 \\
\hline
\end{tabular}

\section{Summary}

Effective geological hazard monitoring method can be used for early warning of geological disasters and is of great significance for the protection of people's property and life safety in the prone areas of geological disasters. In this paper, we first use the GNSS navigation technology to calculate the coordinates of the monitoring points. Then through the coordinate conversion and calculation, the relative position change of the monitoring station to the base station can be obtained. The system has been put into trial operation and the actual running data shows that the geological disaster monitoring method based on GNSS is feasible. The method can provide accurate and real-time measurement data. The results can effectively forecast the geological disasters. GNSS navigation positioning system has 
good scalability and the new monitoring points can be added to the existing system easily, which makes the rapid deployment of monitoring points possible. In addition, the method is a good reference for the design and application of other related systems.

\section{References}

[1] Guo Z W, Li Y H. Design of coordinated control system for beam-carried cranes group based on RTK-GPS. Journal of Beijing University of Aeronautics and Astronautics, 2008, 34(11):1280-1283.

[2] Teng Y L, Shi Y B, Zheng Z. Research on GPS receiver positioning algorithm under bad conditions. Chinese Journal of Scientific Instrument, 2011, 32(8):1887-1884.

[3] Li Shan, Jiang Nan, Huang Jianguo. Underwater GPS positioning algorithm based on randomly distributed buoys. Chinese Journal of Scientific Instrument, 2006, 27(z3):2574-2576.

[4] Zhang J B, Zhang S F, Hu Q. Research on adaptive adjustment of loop gain in GNSS carrier loop. Chinese Journal of Scientific Instrument, 2012, 33(2):293-298.

[5] Wang J P, Gao J X, Liu C. High precision slope deformation monitoring model based on the GPS/Pseudolites technology in open-pit mine. Mining Science and Technology, 2010, 20(1):126-132.

[6] Tang B, Yu X Q, Dong X R. Design intelligent carrier tracking loop based on software GPS receiver. Journal of Beijing University of Aeronautics and Astronautics, 2007, 33(7):807-810.

[7] Liu Y Q, Lian B W, Tang C K. Long baseline high accuracy GNSS relative positioning algorithm. Journal of Northwestern Polytechnical University, 2013, 31(3):451-456.

[8] Tang W M, Sun H X, Chen J. Method of ratio accumulation of GNSS and fast double-differenced ambiguity resolution. Journal of Geodesy and Geodynamics, 2008, 28(5):68-72.

[9] Silva I, Pantoja A, Toledo J C. A quality management system for positioning with GNSS technology. Survey Review, 2010, 42(318):397-405.

[10] Pan X, Nie Y P. Carrier-Aided GPS relative dynamic tracking algorithm. Transactions of Beijing Institute of Technology, 2011, 31(5):594-597.

[11] Bian H F, Zhang S B, Zhang Q Z. On GNSS positional error transformation between common coordinate systems. Journal of Geodesy And Geodynamics, 2012, 32(4):83-86.

[12] Li Y Q, Jiang J, Chen W. Research on real-time altitude monitoring of portal crane based on GNSS-RTK.http://www.controleng.com/single-article/research-on-real-time-altitude-monitoring-of -portal-crane-based-on-gnss-rtk/5317f47748886dd054a7826993667a05.html 